\title{
Article \\ Reductions of Invariant bi-Poisson Structures and Locally Free Actions
}

\author{
Ihor Mykytyuk 10
}

check for updates

Citation: Mykytyuk, I. Reductions of Invariant bi-Poisson Structures and Locally Free Actions. Symmetry 2021, 13, 2043. https://doi.org/10.3390/ sym13112043

Academic Editors: Anatolij K. Prykarpatski and Alexander A. Balinsky

Received: 28 September 2021

Accepted: 20 October 2021

Published: 30 October 2021

Publisher's Note: MDPI stays neutral with regard to jurisdictional claims in published maps and institutional affiliations.
Department of Applied Mathematics, Faculty of Computer Science and Telecommunications, Cracow University of Technology, Warszawska 24, 31155 Cracow, Poland; ihor.mykytyuk@pk.edu.pl ; Tel.: +48-692-136-021

\begin{abstract}
Let $\left(X, G, \omega_{1}, \omega_{2},\left\{\eta^{t}\right\}\right)$ be a manifold with a bi-Poisson structure $\left\{\eta^{t}\right\}$ generated by a pair of $G$-invariant symplectic structures $\omega_{1}$ and $\omega_{2}$, where a Lie group $G$ acts properly on $X$. We prove that there exists two canonically defined manifolds $\left(R_{L^{i}}, G^{i}, \omega_{1}^{i}, \omega_{2}^{i},\left\{\eta_{i}^{t}\right\}\right), i=1,2$ such that (1) $R_{L^{i}}$ is a submanifold of an open dense subset $X_{(H)} \subset X$; (2) symplectic structures $\omega_{1}^{i}$ and $\omega_{2}^{i}$, generating a bi-Poisson structure $\left\{\eta_{i}^{t}\right\}$, are $G^{i}$ - invariant and coincide with restrictions $\left.\omega_{1}\right|_{R_{L^{i}}}$ and $\left.\omega_{2}\right|_{R_{L^{i}}} ;$ (3) the canonically defined group $G^{i}$ acts properly and locally freely on $R_{L^{i}} ;(4)$ orbit spaces $X_{(H)} / G$ and $R_{L^{i}} / G^{i}$ are canonically diffeomorphic smooth manifolds; (5) spaces of G-invariant functions on $X_{(H)}$ and $G^{i}$-invariant functions on $R_{L^{i}}$ are isomorphic as Poisson algebras with the bi-Poisson structures $\left\{\eta^{t}\right\}$ and $\left\{\eta_{i}^{t}\right\}$ respectively. The second Poisson algebra of functions can be treated as the reduction of the first one with respect to a locally free action of a symmetry group.
\end{abstract}

Keywords: bi-Poisson structure; reduction; proper action

MSC: 53D17; 37J15

\section{Introduction}

Two Poisson structures, $\eta_{1}$ and $\eta_{2}$, are said to be compatible if the sum $\eta_{1}+\eta_{2}$, or, equivalently, any linear combination $\eta^{t}=t_{1} \eta_{1}+t_{2} \eta_{2}, t=\left(t_{1}, t_{2}\right) \in \mathbb{R}^{2}$ is a Poisson structure. The family $\left\{\eta^{t}\right\}$ is called a bi-Poisson structure.

The formalism of bi-Poisson (or bi-Hamiltonian in other terminology) structures plays an important role in the theory of integrable systems since it is simultaneously a tool for constructing integrals in involution and studing their completeness, singularities, etc. [1-5]. It turns out that there are two classes of bi-Hamiltonian structures of principally different natures (on the level of local geometry as well as in applications to integrable systems). The bi-Poisson structures of the first class, called bisymplectic, are generated by pairs $\eta_{1}, \eta_{2}$ such that in the pencil $\left\{\eta^{t}\right\}$ almost all members are nondegenerate Poisson structures, i.e., inverse to symplectic forms. Contrastingly, in the pencils corresponding to the second class of Kronecker bi-Poisson structures all the members are degenerate of the same rank [6]. The latter proved to be most effective for constructing the families of commuting functions (which are simply the Casimir functions of generic Poisson structures from the pencil $\left\{\eta^{t}\right\}$ ) and for showing their completeness (which is guaranteed by the above mentioned constancy rank condition [4]).

In [7] A. Panasyuk developed a method of constructing integrable systems based on Poisson reduction of a bi-symplectic G-invariant bi-Poisson structure by means of a Hamiltonian (with respect to both the symplectic forms) action of a Lie group $G$ to a Kronecker bi-Poisson structure. A crucial role in [7] for the checking of the constancy rank condition is played by the fact that the Hamiltonian action of $G$ is locally free. In this case the corank of the generic Poisson structure in the reduced pencil is independent of the parameter and coincides with the index of the Lie algebra $\mathfrak{g}$ (this fact is guaranteed by the so-called inertia lemma from the theory of Hamiltonian actions relating the image of the moment map to the generic stabilizer ([8], Lemma 2.1). In [9] the method of [7] was 
extended to the case when the action of $G$ is not locally free by means of another type of reduction which essentially consists in passing to the so-called Dirac brackets ([10], Section 8.5) of special type.

In this paper we study this reduction procedure in full generality. Namely, we consider the problem of reduction of a bi-Poisson structure $\left\{\eta^{t}\right\}$, which is generated by two symplectic structures, $G$-invariant with respect to a proper action of a Lie group $G$ on a connected manifold $X$ to a bi-Poisson structure $\left\{\widetilde{\eta}^{t}\right\}$ which is $\widetilde{G}$-invariant with respect to a proper locally free action of a Lie group $\widetilde{G}$ on a submanifold $\widetilde{X} \subset X$ (Theorem 1 ). Moreover, the submanifold $(\widetilde{X}, \widetilde{G})$ is very special, allowing to canonically identify the spaces $A^{G}$ of $G$-invariant functions on $X$ and $A^{\widetilde{G}}$ of $\widetilde{G}$-invariant functions on $\widetilde{X}$ and therefore the bi-Poisson structure $\left\{\left(\eta^{t}\right)^{\prime}\right\}$ induced on $A^{G} \simeq A^{\widetilde{G}}$ can be treated as the reduction with respect to a locally free action of a Lie group. As a result, in the case when the action of the Lie group is Hamiltonian with respect to both the symplectic forms (which is most interesting for applications) one can use the machinery of the moment map and the inertia lemma for checking whether $\left\{\left(\eta^{t}\right)^{\prime}\right\}$ is Kronecker or not.

Note that, given a symplectic form $\omega$ on a manifold $X$ and a symplectic submanifold $\widetilde{X} \subset X$, the Poisson bracket related to the Poisson structure $\widetilde{\eta}=\left(\left.\omega\right|_{\tilde{X}}\right)^{-1}$ is an example of a Dirac bracket. In general, if two Poisson structures $\omega_{1}^{-1}$ and $\omega_{2}^{-1}$ are compatible, the Dirac brackets $\left(\left.\omega_{1}\right|_{\widetilde{X}}\right)^{-1},\left(\left.\omega_{2}\right|_{\widetilde{X}}\right)^{-1}$ need not be so (here $\widetilde{X}$ is a symplectic submanifold with respect to both $\left.\left.\omega_{1}\right|_{\widetilde{X}},\left.\omega_{2}\right|_{\widetilde{X}}\right)$. In this paper we deal with a very special situation, when $\left(\left.\omega_{1}\right|_{\tilde{X}}\right)^{-1},\left(\left.\omega_{2}\right|_{\widetilde{X}}\right)^{-1}$ are compatible, which is a consequence of the G-invariance and the special choice of the submanifold $\widetilde{X}$. This situation can be described in more detail as follows.

Given a proper action of a connected Lie group $G$ on a connected manifold $X$ and an isotropy subgroup $H \subset G$ representing the principal orbit type, consider the subset $X_{(H)}$ of $X$ consisting of the points in $X$ with the stabilizer conjugated to $H$ in $G$. Since the manifold $X$ is connected, the subset $X_{(H)}$ is connected, open and dense in $X$ [11]. We consider two subsets $R_{L}$ of $X_{(H)}$ consisting of the points in $X_{(H)}$ with the stabilizer group containing the subgroup $L \subset H \subset G$, where either $L=H$ or $L$ is the identity component $H^{0}$ of $H$. The set $R_{L}$ is a smooth embedded submanifold of $X$. The subgroup $N(L) \subset G$, the normalizer group of $L$ in $G$, acts on $R_{L}$ and the action of the quotient group $N(L) / L$ on $R_{L}$ is locally free and proper.

Let $\omega_{1}$ and $\omega_{2}$ be two $G$-invariant symplectic structures on $X$ determining a bi-Poisson structure $\left\{\eta^{t}\right\}$ on $X$. We show that $R_{L}$ is a symplectic submanifold for an arbitrary Ginvariant symplectic structure on $X$, in particular, the forms $\left.\omega_{1}\right|_{R_{L}}$ and $\left.\omega_{2}\right|_{R_{L}}$ are nondegenerate (if $L=H$ this fact is well known [12]). Then we prove that the symplectic structures $\left.\omega_{1}\right|_{R_{L}}$ and $\left.\omega_{2}\right|_{R_{L}}$ are Poisson compatible, i.e., generate an $N(L) / L$-invariant bi-Poisson structure $\left\{\widetilde{\eta}^{t}\right\}$ on $R_{L}$ (Theorem 1). Due to the isomorphism $X_{(H)} / G=R_{L} /(N(L) / L)$, the second quotient space is a smooth manifold. As a result the sets $A^{G}$ of $G$-invariant functions on $X_{(H)}$ and $A^{\widetilde{G}}$ of $\widetilde{G}$-invariant functions on $R_{L}$, where $\widetilde{G}=N(L) / L$, can be canonically identified. The bi-Poisson structures $\left\{\eta^{t}\right\}$ and $\left\{\widetilde{\eta}^{t}\right\}$ restricted to the space $A^{G} \simeq A^{\widetilde{G}}$ determine the same bi-Poisson structure (Theorem 1).

Since the proper action of the group $N(L) / L$ on the manifold $R_{L}$ is locally free, for investigation of the algebraic properties of the bi-Poisson algebra $A^{G} \simeq A^{\widetilde{G}}$ we can use methods developed in [7] for locally free actions.

We illustrate the theory by a class of examples of reductions of bi-Poisson structures on cotangent bundles to coadjoint orbits (homogeneous spaces) $G / K$, where a compact Lie group $G$ acts on $G / K$ and then on the cotangent bundle $T^{*}(G / K)$ by the lifted action (see Section 3). Here $\omega_{1}$ is the canonical symplectic form $\Omega$ on the cotangent bundle and $\omega_{2}$ is equal to the sum of $\Omega$ and the pull-back of the Kirillov-Kostant-Souriau form. In particular, we describe the submanifolds $X_{(H)}$ and $R_{L}$ and the reduced bi-Poisson structure $\left\{\widetilde{\eta}^{t}\right\}$ on $R_{L}$ (see Proposition 1 and its proof). 
These examples of bi-Poisson structures first appeared in our paper [9] (note that the proofs of the results of Section 3 are new and independent of those from [9]), where they served as a tool in the proof of the complete integrability for geodesic flows of some metrics. The present paper arose from our attempt to understand the general principle standing behind the examples mentioned. The results of this paper are also intended as a tool which can be effectively applied to the study of complete integrability of similar systems, however, such a study lies beyond the scope of this paper since we hope that the results of the present paper are of interest on their own. Note also that the results of this paper are generalizations of results of our preprint [13].

The paper is organized as follows. It is divided into three sections and Appendix A among which Section 2 is the principal one. The main result of the paper, Theorem 1, is contained in Section 2.3, while Sections 2.1-2.2 are introductory ones. They contain definitions and general results needed for the formulation and proof of our main result (its crucial ingredients are Lemmas 2 and 3). Section 3 contains the above mentioned examples. In Appendix A we formulate and prove one statement (Lemma A1) from the general theory of Lie groups which is used in the introductory considerations of Section 2.2.

\section{Proper Actions of Lie Groups and Reductions of Invariant bi-Poisson Structures}

Let $G$ be a connected Lie group acting properly on a smooth connected manifold $X$. For any point $x \in X$ denote by $G_{x}$ its isotropy group. We remark that the group $G_{x}$ is compact because the action of $G$ on $X$ is proper.

For any Lie group $A \subset G$ we will denote by $A^{0}$ its connected component of the identity element (the identity component for short) and by $N(A)$, the normalizer group of $A$ in $G$.

Fix some isotropy subgroup $H \subset G$ determining the principal orbit type. In this case the subset

$$
X_{(H)}=\left\{x \in X: G_{x}=g H^{-1} \text { for some } g \in G\right\}
$$

of $X$, consisting of all orbits $G \cdot x$ in $X$ isomorphic to $G / H$, is an open and dense subset of $X$ (see ([11], Section 2.8 and Th. 2.8.5)). The open submanifold $X_{(H)} \subset X$ is G-invariant by definition. It is well known that the orbit space $X_{(H)} / G$ is a smooth manifold. Mainly to fix the notation we shall prove this fact below.

Consider the subset

$$
X_{H}=\left\{x \in X: G_{x}=H\right\}
$$

of $X$ consisting of the points in $X$ with stabilizer precisely equal to $H$. It is clear that $X_{H} \subset X_{(H)}$. The set $X_{H}$ is a smooth embedded submanifold of $X$ ([12], Prop. 2.4.7). It is easy to see that the normalizer group $N(H)$ of $H$ in $G$ acts on $X_{H}$ and that every G-orbit in $X_{(H)}$ intersects $X_{H}$ on an $N(H)$ orbit. Furthermore, the quotient group $N(H) / H$ acts freely on $X_{H}$ and generates the same orbit space. This action of $N(H) / H$ is proper because the subgroup $N(H) \subset G$ is closed. Therefore $X_{H} /(N(H) / H)$ is a smooth manifold ([14], Ch. 3, Section 1.5, Prop. 10) and, consequently, due to orbit isomorphism

$$
X_{(H)} / G \simeq X_{H} /(N(H) / H)
$$

$X_{(H)} / G$ is also a smooth manifold.

\subsection{The Submanifold $R_{L}$ of the Single Orbit Type Submanifold $X_{(H)}$}

Let $L \in\left\{H, H^{0}\right\}$, where we recall that $H^{0}$ is the identity component of $H$. Let $\mathfrak{g}$ be the Lie algebra of the Lie group $G$. Denote by $\mathfrak{h}$ and $\mathfrak{n}(L)$ the Lie algebras of the Lie group $H$ and of the normalizer group $N(L)$ of $L$ in $G$ respectively. By definition

$$
N(H) \subset N(L) \subset N\left(H^{0}\right) \text { and } \mathfrak{n}(H) \subset \mathfrak{n}(L) \subset \mathfrak{n}\left(H^{0}\right) .
$$

Since the Lie subgroup $\operatorname{Ad}(H)$ of $\operatorname{Ad}(G)$ is compact, there is an $\operatorname{Ad}(H)$-invariant scalar product $\langle\cdot, \cdot\rangle^{H}$ on the Lie algebra $\mathfrak{g}$. Denote by $\mathfrak{p}=\mathfrak{p}_{L}$ the orthogonal complement to 
$\mathfrak{n}(L)$ in $\mathfrak{g}$ with respect to $\langle\cdot, \cdot\rangle^{H}$. By the $\operatorname{Ad}(L)$-invariance of the form and by the inclusion $L \subset N(L)$ we have that

$$
\mathfrak{g}=\mathfrak{p} \oplus \mathfrak{n}(L), \quad \operatorname{Ad}(L)(\mathfrak{n}(L))=\mathfrak{n}(L), \quad \operatorname{Ad}(L)(\mathfrak{p})=\mathfrak{p} .
$$

Let

$$
R_{L}=\left\{x \in X_{(H)}: L \subset G_{x}\right\},
$$

where we recall that $G_{x}$ stands for the isotropy group $G_{x}$ of $x$.

It is clear that $R_{H}=X_{H}, R_{H} \subset R_{H^{0}}$ and the set $R_{H^{0}}$ is a set of all $x \in X_{(H)}$ such that the Lie algebras of $G_{x}$ and $H$ coincide, i.e., $G_{x}^{0}=H^{0}$. We will prove below that $R_{L}$ is an embedded submanifold of $X_{(H)}$ and $\operatorname{dim} R_{H^{0}}-\operatorname{dim} R_{H}=\operatorname{dim} \mathfrak{n}\left(H^{0}\right)-\operatorname{dim} \mathfrak{n}(H)$.

Lemma 1. The set $R_{L}$ is an embedded submanifold of the manifold $X_{(H)}$ and for any $x \in R_{L}$ the tangent space $T_{x} R_{L}$ is given by

$$
T_{x} R_{L}=\left\{v \in T_{x} X_{(H)}: h_{*}(x)(v)=v, \forall h \in L\right\} .
$$

The quotient group $N(L) / L$ acts locally freely on the set $R_{L}$ if $L=H^{0}$ and freely if $L=H$ and the orbit space

$$
R_{L} /(N(L) / L) \simeq X_{(H)} / G
$$

is a smooth manifold.

Proof. We will prove the lemma only in the case when $L=H^{0}$ because in the case $L=H$ the lemma follows immediately from ([12], Prop. 2.4.7).

To prove the lemma we will use the method from ([12], Ch.2, Sections 2.3 and 2.4). Due to the fact that $X_{(H)}$ is a single orbit type manifold, the local description of the $G$-action on this connected manifold is very simple. For the point $x \in X_{(H)}$ there is a $G$-invariant open neighborhood $O(x)$ in $X_{(H)}$ and a G-equivariant diffeomorphism $\phi: G / G_{x} \times W \rightarrow O(x)$, where $G$ acts naturally on $G / G_{x}$ and trivially on $W$ ([12], Th.2.3.28). Here the cross-section $W$ is an open ball around 0 in some real linear space $\mathbb{R}^{k}$ (of dimension $\left.k=\operatorname{dim} X-\operatorname{dim}\left(G / G_{x}\right)\right)$ and $\phi(o, 0)=x$, where $o=G_{x} \in G / G_{x}$.

Since $x \in R_{H^{0}} \subset X_{(H)}$, we have $G_{x}^{0}=H^{0}$. Under the above mentioned $\phi$-identification of the open neighborhood $O(x), x \in R_{H^{0}}$, with $G / G_{x} \times W$ the subset $O_{H^{0}}(x)=O(x) \cap R_{H^{0}}$ is $\phi$-isomorphic to $N\left(H^{0}\right) / G_{x} \times W \subset G / G_{x} \times W$, where $N\left(H^{0}\right) / G_{x}$ is considered as a closed embedded submanifold of $G / G_{x}$ ([12], Prop. 2.4.6). From this local description it follows that $R_{H^{0}}$ is a (locally closed) embedded submanifold of $X_{(H)}$ and the submanifold $\mathrm{O}_{H^{0}}(x)$ is $\mathrm{N}\left(H^{0}\right)$-invariant.

Let us prove relation (7). The group $H^{0}$ acts on $W$ trivially and the tangent action of $H^{0}$ on the tangent space $\mathfrak{g} / \mathfrak{h}=T_{o}\left(G / G_{x}\right)$ is induced by the $\operatorname{Ad}\left(H^{0}\right)$-action on $\mathfrak{g}$. Let $\mathfrak{\xi} \in \mathfrak{g}$. By Formula (A1) from Appendix, $\operatorname{Ad}(h)(\xi+\mathfrak{h})=\xi+\mathfrak{h}$ for all $h \in H^{0}$ if and only if $\xi \in \mathfrak{n}\left(H^{0}\right)$. Taking into account that $\mathfrak{n}\left(H^{0}\right) / \mathfrak{h}=T_{o}\left(N\left(H^{0}\right) / G_{x}\right)$, we obtain that

$$
T_{(o, 0)}\left(N\left(H^{0}\right) / G_{x} \times W\right)=\left\{v \in T_{(o, 0)}\left(G / G_{x} \times W\right): h_{*}(o, 0)(v)=v, \forall h \in H^{0}\right\} .
$$

Hence, since the diffeomorphism $\phi$ is $G$-equivariant, we get (7).

Since for each $x \in R_{H^{0}} \subset X_{(H)}$ its isotropy group $G_{x}$ is conjugated to $H$ in $G$, it is easy to check that

(1) The subgroup $N\left(H^{0}\right)$ acts on $R_{H^{0}}$ and $H^{0} \subset N\left(H^{0}\right)$ acts trivially on $R_{H^{0}}$;

(2) Every G-orbit in $X_{(H)}$ intersects $R_{H^{0}}$ on an $N\left(H^{0}\right)$-orbit;

(3) $N\left(H^{0}\right) \cdot X_{H}=R_{H^{0}}$ (if $G_{x}^{0}=H^{0}$ and $g G_{x} g^{-1}=H$, then $g \in N\left(H^{0}\right)$ ).

The quotient group $N\left(H^{0}\right) / H^{0}$ acts locally freely on $R_{H^{0}}$ (with finite isotropy group $G_{x} / H^{0} \simeq H / H_{0}$ at $x \in R_{H^{0}}$ ) and generates the same orbit space as $G$ on $X_{(H)}$. This action of $N\left(H^{0}\right) / H^{0}$ is proper because the subgroup $N\left(H^{0}\right) \subset G$ is closed. Since by relation (3) 
$X_{(H)} / G$ is a smooth manifold, the quotient space $R_{H^{0}} /\left(N\left(H^{0}\right) / H^{0}\right) \simeq X_{(H)} / G$ is also a smooth manifold.

\subsection{The Local Structure of the Single Orbit Type Submanifold $\mathbf{X}_{(\mathbf{H})}$ Near $\mathbf{R}_{\mathbf{L}}$}

The action of $G$ determines a linear map $\xi \mapsto \xi_{X}$, where $\xi_{X}$ denotes the vector field on $X$ generated by one-parameter subgroup $\exp t \xi \subset G$. For any subspace $\mathfrak{a} \subset \mathfrak{g}$ and a point $x \in X$ put $\mathfrak{a}(x) \stackrel{\text { def }}{=}\left\{\xi_{X}(x): \xi \in \mathfrak{a}\right\}$.

In this subsection we describe a canonical complementary subbundle $\mathcal{P}=\mathcal{P}_{L}$ to $T R_{L}$ in $\left.T X_{(H)}\right|_{R_{L}}$ for which the splitting $\left.T X_{(H)}\right|_{R_{L}}=\mathcal{P} \oplus T R_{L}$ is orthogonal with respect to an arbitrary $G$-invariant nondegenerate form on $X$. The existence of such a canonical subbundle $\mathcal{P}$ determines a local structure of $X_{(H)}$ near $R_{L}$.

Choose some point $x \in R_{L} \subset X_{(H)}$. Due to the compactness of the Lie subgroup $\operatorname{Ad}\left(G_{x}\right)$ of $\operatorname{Ad}(G)$ there exists an $\operatorname{Ad}\left(G_{x}\right)$-invariant scalar product $\langle\cdot, \cdot\rangle^{G_{x}}$ on the Lie algebra $\mathfrak{g}$. Denote by $\mathfrak{p}^{x}=\mathfrak{p}_{L}^{x}$ the orthogonal complement to $\mathfrak{n}(L)$ in $\mathfrak{g}$ with respect to $\langle\cdot, \cdot\rangle^{G_{x}}$. Since $L \subset N(L)$ and the form is always $\operatorname{Ad}(L)$-invariant (either $G_{x}=H=L$ or $G_{x}^{0}=H^{0}=L$ ), we have

$$
\mathfrak{g}=\mathfrak{p}^{x} \oplus \mathfrak{n}(L), \quad \operatorname{Ad}(L)(\mathfrak{n}(L))=\mathfrak{n}(L), \quad \operatorname{Ad}(L)\left(\mathfrak{p}^{x}\right)=\mathfrak{p}^{x} .
$$

Identifying the tangent space to the homogeneous space $G / G_{x}$ at $o=G_{x} \in G / G_{x}$ with the orthogonal complement $\mathfrak{h}^{\perp x} \subset \mathfrak{g}$ to $\mathfrak{h}$ in $\mathfrak{g}$ with respect to $\langle\cdot, \cdot\rangle^{G_{x}}$, we obtain that $\mathfrak{p}^{x} \subset \mathfrak{h}^{\perp x}$ is a complementary subspace to the tangent space $T_{o}\left(N(L) / G_{x}\right)$ in $T_{o}\left(G / G_{x}\right)$. Using our G-equivariant identification $\phi: G / G_{x} \times W \rightarrow O(x), \phi(0,0)=x$, we conclude that the space $\mathfrak{p}^{x}(x) \stackrel{\text { def }}{=}\left\{\xi_{X}(x), \xi \in \mathfrak{p}^{x}\right\}$ is a complementary subspace to $T_{x} R_{L}$ in $T_{x} X_{(H)}$.

The $\operatorname{Ad}\left(G_{x}\right)$-invariant scalar product $\langle\cdot, \cdot\rangle^{G_{x}}$ on $T_{o}\left(G / G_{x}\right)=\mathfrak{h}^{\perp x}$ and any scalar product on $T_{0} W$ determine an $G_{x}$-invariant scalar product on the tangent space $T_{0}\left(G / G_{x}\right) \oplus$ $T_{0} W$. Now using the $G$-equivariant diffeomorphism $\phi$ we obtain the $G_{x}$-invariant scalar product $\langle\cdot, \cdot\rangle_{x}$ on the space $T_{x} X_{(H)}$ at $x=\phi(o, 0)$ such that $\left\langle\mathfrak{p}^{x}(x), T_{x} R_{L}\right\rangle_{x}=0$. In general, $\mathfrak{p}^{x} \neq \mathfrak{p}$ (the subspace $\mathfrak{p}$ was defined in Section 2.1) but

$$
\mathfrak{p}^{x} \oplus \mathfrak{h}=\mathfrak{p} \oplus \mathfrak{h} .
$$

The proof of this identity is given in Appendix A (see Lemma A1). Now, taking into account that $\mathfrak{h}$ is the isotropy algebra of the point $x \in R_{L}$ (either $G_{x}^{0}=H^{0}$ or $G_{x}=H$ ), i.e., $\mathfrak{h}(x)=0$, we obtain that $\mathfrak{p}^{x}(x)=\mathfrak{p}(x)$. Thus the space

$$
\mathcal{P}(x) \stackrel{\text { def }}{=} \mathfrak{p}(x)=\left\{\xi_{X}(x), \xi \in \mathfrak{p}\right\}, \quad x \in R_{L},
$$

is the orthogonal complement to the tangent space $T_{x} R_{L}$ in $T_{x} X_{(H)}$ :

$$
T_{x} X_{(H)}=\mathcal{P}(x) \oplus T_{x} R_{L}, \quad\left\langle\mathcal{P}(x), T_{x} R_{L}\right\rangle_{x}=0, \quad x \in R_{L} .
$$

We will show below that the space $\mathcal{P}(x)$ is the orthogonal complement to the space $T_{x} R_{L}$ in $T_{x} X_{(H)}$ with respect to any $G_{x}$-invariant nondegenerate bi-linear form on $T_{x} X_{(H)}$.

Let $x \in R_{L}$. Since $h_{*}\left(\xi_{X}\right)=(\operatorname{Ad}(h)(\xi))_{X}$ for any $\xi \in \mathfrak{g}, h \in G, \operatorname{Ad}(L)(\mathfrak{p})=\mathfrak{p}$ and $L \cdot x=x$ by definition of $R_{L}$ (always $L \subset G_{x}$ ), the space $\mathcal{P}(x)$ is $L$-invariant, i.e., $h_{*}(x)(\mathcal{P}(x))=\mathcal{P}(x)$ for any $h \in L \subset H$. It is evident that the union $\mathcal{P}=\bigcup_{x \in R_{L}} \mathcal{P}(x)$ is a trivial vector bundle over $R_{L}$ and $T X_{(H)} \mid R_{L}=\mathcal{P} \oplus T R_{L}$. The vector fields $\left.\xi_{X}\right|_{R_{L}}, \xi \in \mathfrak{p}$, are global sections of $\mathcal{P}$.

Lemma 2. Let $\alpha(x)$ be a $G_{x}$-invariant nondegenerate bi-linear form on the space $T_{x} X_{(H)}, x \in R_{L}$. Then $\alpha(x)\left(\mathcal{P}(x), T_{x} R_{L}\right)=0$, i.e., $\mathcal{P}(x)$ is the orthogonal complement to the space $T_{x} R_{L}$ in $T_{x} X_{(H)}$ with respect to the form $\alpha(x)$ and the restrictions $\left.\alpha(x)\right|_{\mathcal{P}(x)},\left.\alpha(x)\right|_{T_{x} R_{L}}$ are nondegenerate.

Proof. To prove that $\alpha(x)\left(\mathcal{P}(x), T_{x} R_{L}\right)=0$ we will use the method of the proof of Lemma 27.1 in [15]. We have shown that there exists a $G_{x}$-invariant scalar product $\langle\cdot, \cdot\rangle_{x}$ on 
the space $T_{x} X_{(H)}=\mathcal{P}(x) \oplus T_{x} R_{L}$ such that Formula (12) holds. The form $\alpha(x)$ is $G_{x^{-}}$ invariant with respect to the tangent action $h_{*}(x): T_{x} X_{(H)} \rightarrow T_{x} X_{(H)}$ of the group $G_{x}$. Thus there exists a unique nondegenerate linear map $J: T_{x} X_{(H)} \rightarrow T_{x} X_{(H)}$ such that $\alpha(x)(u, v)=\langle u, J v\rangle_{x}$ for all $u, v \in T_{x} X_{(H)}$ and $J \cdot h_{*}(x)=h_{*}(x) \cdot J$ for all $h \in G_{x} \supset L$. By (7) the subspace $T_{x} R_{L} \subset T_{x} X_{(H)}$ is the set of all $L$-fixed vectors in $T_{x} X_{(H)}$. Now we get the inclusion $J\left(T_{x} R_{L}\right) \subset T_{x} R_{L}$ due to the fact that $J$ commutes with the $L$-action on $T_{x} X_{(H)}$. Thus $\alpha(x)\left(\mathcal{P}(x), T_{x} R_{L}\right)=\left\langle\mathcal{P}(x), J\left(T_{x} R_{L}\right)\right\rangle_{x}=0$ by (12). Since $T_{x} X_{(H)}=\mathcal{P}(x) \oplus T_{x} R_{L}$ and the form $\alpha(x)$ is nondegenerate, we obtain the last assertion of the lemma.

The following lemma asserts the existence of local coordinate systems in the manifold $X_{(H)}$ (of dimension $m$ ) near the submanifold $R_{L}$ (of codimension $p=p_{L}$ in $X_{(H)}$ ) consistent with any $G$-invariant nondegenerate bi-linear form on $X$.

Lemma 3. For each point $x \in R_{L}$ there exists an open subset $U(x) \subset X_{(H)}$ and a coordinate system $\left(U(x), y_{1}, \ldots, y_{p}, y_{p+1}, \ldots, y_{m}\right)$, in $X_{(H)}$ around the point $x$ such that

(1) All coordinates of the point $x$ vanish: $y_{1}(x)=\ldots=y_{m}(x)=0$;

(2) The subset $U_{L}(x)=U(x) \cap R_{L}$ of $U(x)$ is the set $\left\{z \in U(x): y_{1}(z)=\ldots=y_{p}(z)=0\right\}$;

(3) The vectors $\partial / \partial y_{i}, i=1, \ldots, p$, and the vectors $\partial / \partial y_{j}, j=p+1, \ldots, m$, at a point $z \in U_{L}(x)$ span the spaces $\mathcal{P}(z)$ and $T_{z} R_{L}$ respectively;

(4) Any G-invariant nondegenerate bi-linear form $\alpha$ on $X$ at a point $z \in U(x)$ in the corresponding basis $\left\{\partial / \partial y_{1}, \ldots, \partial / \partial y_{m}\right\}, \quad$ has the matrix $\left(\begin{array}{ll}A(y(z)) & B(y(z)) \\ C(y(z)) & D(y(z))\end{array}\right)$ such that $B(y(z))=C(y(z))=0$ and the matrices $A(y(z))$, $D(y(z))$ are nondegenerate for $z \in U_{L}(x)$.

Proof. Recall that the group $N(L)$ is a closed subgroup of $G$ because $L$ by definition is also closed in $G$ (either $L=H$ or $L=H^{0}$ ). Additionally, we have the $\operatorname{Ad}(L)$-invariant splitting $\mathfrak{g}=\mathfrak{p} \oplus \mathfrak{n}(L)$ of $\mathfrak{g}$ (see Formula (5)). Therefore for some open $\operatorname{Ad}(L)$-invariant ball $Y$ around 0 in $\mathfrak{p}$ the map

$$
Y \times N(L) \rightarrow G, \quad(y, n) \mapsto \exp y \cdot n,
$$

is a $L$-equivariant diffeomorphism onto the open neighborhood of the identity element in $G$. This map intertwines the action $h \cdot(y, n)=(\operatorname{Ad}(h)(y), h n)$ of $L$ on $Y \times N(L)$ and the left action of $L$ on $G$. Thus the map

$$
Y \times N(L) / G_{x} \rightarrow G / G_{x}, \quad\left(y, n G_{x}\right) \mapsto(\exp y \cdot n) G_{x}
$$

is a $L$-equivariant diffeomorphism onto the open neighborhood of the point $o=G_{x}$ in $G / G_{x}$ and, consequently, the map

$$
Y \times N(L) / G_{x} \times W \rightarrow \phi\left(G / G_{x} \times W\right)=O(x),\left(y, n G_{x}, w\right) \mapsto \phi\left(\exp y \cdot n G_{x}, w\right)
$$

is an $L$-equivariant diffeomorphism onto the open $L$-invariant neighborhood $O_{1}(x) \subset O(x)$ in $X_{(H)}$ containing the neighborhood $O_{L}(x)=\phi\left(N(L) / G_{x} \times W\right)$ of $x$ in $R_{L}$. Here the action of $L$ on $Y \times N(L) / G_{x} \times W$ is induced by the action of $L$ on $Y \times N(L)$, i.e., $h \cdot\left(y, n G_{x}, w\right)=$ $\left(\operatorname{Ad}(h)(y), h n G_{x}, w\right)$ for $h \in L$. By the G-equivariance of $\phi$, the map

$$
\psi: Y \times O_{L}(x) \rightarrow O_{1}(x), \quad(y, z) \mapsto(\exp y) \cdot z
$$

is also a diffeomorphism such that $\psi(0, z)=z$ for all $z \in O_{L}(x)$. Moreover, $\psi_{*(0, z)}\left(T_{0} Y, 0\right)=$ $\mathcal{P}(z)$ for $z \in O_{L}(x)$ because by $(11) \mathcal{P}(z)=\left\{\xi_{X}(z), \xi \in \mathfrak{p}\right\}$ and $Y \subset \mathfrak{p}$. This diffeomorphism $\psi$ is $L$-equivariant with respect to the action $h \cdot(y, z)=(\operatorname{Ad}(h)(y), h \cdot z)$ of $L$ on $Y \times O_{L}(x)$ and the $L$-action on $O_{1}(x) \subset X_{(H)}$. 
The existence of the diffeomorphism $\psi$ means in particular that there exists a coordinate system $\left(U(x), y_{1}, \ldots, y_{p}, y_{p+1}, \ldots, y_{m}\right)$ in $X_{(H)}$ around the point $x \in R_{L}$ with properties (1)-(3).

Let us prove property (4) for this coordinate system. Since $h \cdot x=x$ for all $h \in G_{x}$, the nondegenerate form $\alpha(x)$ is $G_{x}$-invariant with respect to the tangent action $h_{*}(x): T_{x} X \rightarrow$ $T_{x} X$ of the group $G_{x}$. Then $\alpha(x)\left(\mathcal{P}(x), T_{x} R_{L}\right)=0$ and the restrictions $\left.\left.\alpha(x)\right|_{\mathcal{P}(x)} \alpha(x)\right|_{T_{x} R_{L}}$ are nondegenerate in view of Lemma 2 . Therefore by property (3) of the coordinate system under consideration around the point $x$ the matrices $B(y(z)), C(y(z))$ vanish and the matrices $A(y(z)), D(y(z))$ are nondegenerate for any $z \in U_{L}(x)$.

\subsection{The Principal Orbit Type Submanifold $X_{(H)}$ and Reduced bi-Poisson Structures on $R_{L}$}

Here as before $X_{(H)}$ is a principal orbit type submanifold of $X$. We will use the notation introduced in the previous Sections 2.1 and 2.2. Denote by $\mathcal{E}(M)$ the space of smooth functions on a manifold $M$.

Let $\eta$ be a $G$-invariant Poisson structure on the manifold $X$. Put $A^{G} \subset \mathcal{E}\left(X_{(H)}\right)$ for the set of all $G$-invariant functions on the open submanifold $X_{(H)} \subset X$. By the $G$-invariance of $\eta$, the space $A^{G}$ is a Poisson subalgebra of $\left(\mathcal{E}\left(X_{(H)}\right), \eta\right)$. The structure $\eta$ determines a Poisson structure on the smooth manifold (see (8))

$$
\mathbf{X}=X_{(H)} / G \simeq R_{L} /(N(L) / L)
$$

and $A^{G} \simeq \mathcal{E}(\mathbf{X})$. Put $\widetilde{G}=N(L) / L$. Denoting by $\pi_{(H)}: X_{(H)} \rightarrow \mathbf{X}$ and $\pi_{L}: R_{L} \rightarrow \mathbf{X}$ the natural submersions, we obtain two isomorphic Poisson algebras, $A^{G}=\pi_{(H)}^{*}(\mathcal{E}(\mathbf{X}))$ of $G$-invariant functions on $X_{(H)}$ and $A^{\widetilde{G}}=\pi_{\mathfrak{h}}^{*}(\mathcal{E}(\mathbf{X}))$ of $\widetilde{G}$-invariant functions on $R_{L}$, where the second structure is induced by the natural identification $A^{G} \simeq A^{\widetilde{G}}$. On the first algebra $A^{G}$ its bracket is induced by the Poisson structure $\eta$ defined on the whole space $X_{(H)}$. A question arises: is there some Poisson structure on the manifold $R_{L}$ which induces the above mentioned bracket on $A^{\widetilde{G}}$. We will prove that such a Poisson structure exists if the Poisson structure $\eta$ on $X$ is nondegenerate, i.e., $\eta=\omega^{-1}$, where $\omega$ is some $G$-invariant symplectic structure on $X$.

As it follows from Lemma 2 in this case the pair $\left(R_{L}, \widetilde{\omega}\right)$, where, $\widetilde{\omega}=i^{*} \omega$ and $i: R_{L} \rightarrow X$ is the natural embedding, is a symplectic manifold (the restriction $\left.\omega(x)\right|_{T_{x} R_{L}}$ is nondegenerate for all $x \in R_{L}$ ). For any function $f \in A^{G}$ its Hamiltonian vector field $\mathcal{H}_{f}$ is tangent to the submanifold $R_{L}$ at each point $x \in R_{L}$. This easily follows from the fact that $d f\left(\tilde{\zeta}_{X}\right)=0$ for all $\xi \in \mathfrak{g}$ and, in particular, for all $\xi \in \mathfrak{p}$, i.e.,

$$
\omega(x)\left(\mathcal{H}_{f}(x), \mathcal{P}(x)\right) \stackrel{\text { def }}{=}-d f(x)(\mathcal{P}(x))=0 .
$$

As $T_{x} R_{L}$ is a skew-orthogonal complement to $\mathcal{P}(x)$ by Lemma 2 we conclude that $\mathcal{H}_{f}(x) \in T_{x} R_{L}$. Therefore, for any $x \in R_{L}$ and any vector field $Y$ tangent to $R_{L}$ we have

$$
-d\left(i^{*} f\right)(x)(Y(x))=-d f(x)(Y(x))=\omega(x)\left(\mathcal{H}_{f}(x), Y(x)\right)=\widetilde{\omega}(x)\left(\mathcal{H}_{f}(x), Y(x)\right),
$$

i.e., the vector field $\left.\mathcal{H}_{f}\right|_{R_{L}}$ is the Hamiltonian vector field of the function $i^{*} f$ with respect to the form $\widetilde{\omega}$. Moreover, for any functions $f_{1}, f_{2} \in A^{G}$ at $x \in R_{L}$ we get the equality

$$
\begin{aligned}
& \eta(x)\left(d f_{1}(x),\right.\left.d f_{2}(x)\right) \stackrel{\text { def }}{=} \omega(x)\left(\mathcal{H}_{f_{2}}(x), \mathcal{H}_{f_{1}}(x)\right) \\
&=\widetilde{\omega}(x)\left(\mathcal{H}_{i^{*} f_{2}}(x), \mathcal{H}_{i^{*} f_{1}}(x)\right)=\widetilde{\eta}(x)\left(d\left(i^{*} f_{1}\right)(x), d\left(i^{*} f_{2}\right)(x)\right),
\end{aligned}
$$

where $\widetilde{\eta}$ is the Poisson structure $\widetilde{\omega}^{-1}$ on $R_{L}$.

A pair $\left(\eta_{1}, \eta_{2}\right)$ of linearly independent bi-vector fields (bi-vectors for short) on a manifold $X$ is called Poisson if $\eta^{t} \stackrel{\text { def }}{=} t_{1} \eta_{1}+t_{2} \eta_{2}$ is a Poisson bi-vector for any $t=\left(t_{1}, t_{2}\right) \in$ $\mathbb{R}^{2}$, i.e., each bi-vector $\eta^{t}$ determines on $X$ a Poisson structure with the Poisson bracket 
$\{,\}^{t}:\left(f_{1}, f_{2}\right) \mapsto \eta^{t}\left(d f_{1}, d f_{2}\right)$; the whole family of Poisson bi-vectors $\left\{\eta^{t}\right\}_{t \in \mathbb{R}^{2}}$ is called a bi-Poisson structure. Remark here that a pair $\left(\eta_{1}, \eta_{2}\right)$ of linearly independent Poisson structures is Poisson if and only if $t_{1} \eta_{1}+t_{2} \eta_{2}$ is a Poisson bi-vector for some $\left(t_{1}, t_{2}\right) \in \mathbb{R}^{2}$ nonproportional to $(1,0),(0,1)$. Indeed, the bi-vector $\eta^{t}$ is Poisson if and only if $\left[\eta^{t}, \eta^{t}\right]_{S}=0$, where $[,]_{S}$ is the so-called Schouten bracket ([10], Section 10.6). The last equation is quadratic with respect to $t_{1}: t_{2}$.

A bi-Poisson structure $\left\{\eta^{t}\right\}$ (we will often skip the parameter space) can be viewed as a two-dimensional vector space of Poisson bi-vectors, the Poisson pair $\left(\eta_{1}, \eta_{2}\right)$ as a basis in this space. Obviously, if the Poisson structures $\eta_{1}$ and $\eta_{2}$ are $G$-invariant, then these structures induce a bi-Poisson structure on the manifold $\mathbf{X}=X_{(H)} / G \simeq R_{L} /(N(L) / L)$ and, consequently, linear families of brackets on the spaces $\mathcal{E}(\mathbf{X}), A^{G}$, and $A^{\widetilde{G}}$. The theorem below asserts that in a particular case the linear family of brackets on the space $A^{\widetilde{G}}$ is induced by some canonically defined bi-Poisson structure on the manifold $R_{L}$. Note that the action of the group $\widetilde{G}=N(L) / L$ on $R_{L}$ is locally free (free if $L=H$ ).

Theorem 1. Let $\eta_{1}=\omega_{1}^{-1}$ and $\eta_{2}=\omega_{2}^{-1}$, where $\omega_{1}, \omega_{2}$ are some G-invariant symplectic forms on X. Assume that the Poisson structures $\eta_{1}$ and $\eta_{2}$ determine a bi-Poisson structure on X. If the forms $\widetilde{\omega}_{1}=i^{*} \omega_{1}$ and $\widetilde{\omega}_{2}=i^{*} \omega_{2}$ are linearly independent on $R_{L}$ (here $i: R_{L} \rightarrow X$ is the natural embedding), then the Poisson structures $\widetilde{\eta}_{1}=\widetilde{\omega}_{1}^{-1}$ and $\widetilde{\eta}_{2}=\widetilde{\omega}_{2}^{-1}$ determine a $\widetilde{G}$-invariant bi-Poisson structure on $R_{L}$. This bi-Poisson structure induces on the space $A^{\widetilde{G}}=A^{G}$ the same linear family of brackets as the bi-Poisson structure induced by the pair $\left(\eta_{1}, \eta_{2}\right)$ on the space $A^{G}$. The action of the group $\widetilde{G}=N(L) / L$ on $R_{L}$ is locally free.

Proof. It is sufficient to perform local reasoning. Fix some point $x \in R_{L}$ and consider in $X$ the coordinate system $\left(U(x), y_{1}, \ldots, y_{p}, y_{p+1}, \ldots, y_{m}\right)$ around the point $x$ as in Lemma 3 . Then in these coordinates the symplectic forms $\omega_{a}, a=1,2$, are described by the skewsymmetric matrices

$$
W_{a}(y(z))=\left(\begin{array}{cc}
A_{a}(y(z)) & B_{a}(y(z)) \\
C_{a}(y(z)) & D_{a}(y(z))
\end{array}\right), \quad \text { for } z \in U(x),
$$

such that

$$
W_{a}(y(z))=\left(\begin{array}{cc}
A_{a}(y(z)) & 0 \\
0 & D_{a}(y(z))
\end{array}\right), \quad \text { for } z \in U_{L}(x) \subset U(x) .
$$

Recall that $U_{L}(x)=U(x) \cap R_{L}$ and $y_{1}(z)=\ldots=y_{p}(z)=0$ if $z \in U_{L}(x)$. By the definition, the Poisson structure $\eta^{t}=t_{1} \eta_{1}+t_{2} \eta_{2}$ is determined by the $m \times m$-matrix $t_{1} W_{1}^{-1}(y)+t_{2} W_{2}^{-1}(y)$ :

$$
\eta^{t}(y)=\sum_{1 \leqslant i<j \leqslant m}\left(t_{1} W_{1}^{-1}+t_{2} W_{2}^{-1}\right)_{i j}(y) \frac{\partial}{\partial y_{i}} \wedge \frac{\partial}{\partial y_{j}}
$$

Since the Poisson structures $\eta_{1}, \eta_{2}$ are nondegenerate, for some $t=\left(t_{1}, t_{2}\right) \in \mathbb{R}^{2} \backslash$ $((\mathbb{R} \times\{0\}) \cup(\{0\} \times \mathbb{R}))$ the Poisson structure $\eta^{t}$ is nondegenerate at each point of some open neighborhood of the point $x$, which we assume, without loss of generality, to be the original open neighborhood $U(x)$. Then the skew-symmetric matrix $\left(t_{1} W_{1}^{-1}(y)+\right.$ $\left.t_{2} W_{2}^{-1}(y)\right)^{-1}$ is a matrix of some symplectic form $\omega^{t}$ on $U(x)$, i.e., the form

$$
\sum_{1 \leqslant i<j \leqslant m}\left(\left(t_{1} W_{1}^{-1}+t_{2} W_{2}^{-1}\right)^{-1}\right)_{i j}(y) d y_{i} \wedge d y_{j}
$$


is closed. Thus the form $i^{*} \omega^{t}$, where $\left.i\right|_{U_{L}(x)}: U_{L}(x) \rightarrow U(x)$ is the embedding $\left(y_{p+1}, \ldots, y_{m}\right) \mapsto\left(0, \ldots, 0, y_{p+1}, \ldots, y_{m}\right)$ is also closed. By (14) for points $z \in U_{L}(x)$ with coordinates $y=\left(0, \ldots, 0, y_{p+1}, \ldots, y_{m}\right)$ we have

$$
\left(t_{1} W_{1}^{-1}+t_{2} W_{2}^{-1}\right)^{-1}(y)=\left(\begin{array}{cc}
\left(t_{1} A_{1}^{-1}+t_{2} A_{2}^{-1}\right)^{-1}(y) & 0 \\
0 & \left(t_{1} D_{1}^{-1}+t_{2} D_{2}^{-1}\right)^{-1}(y)
\end{array}\right) .
$$
form

Taking into account that $y_{1}(z)=\ldots=y_{p}(z)=0$ on the set $U_{L}(x)$ we obtain that the

$$
\left(i^{*} \omega^{t}\right)(y)=\sum_{p+1 \leqslant i<j \leqslant m}\left(\left(t_{1} D_{1}^{-1}+t_{2} D_{2}^{-1}\right)^{-1}\right)_{i j}(y) d y_{i} \wedge d y_{j},
$$

where $y=\left(0, \ldots, 0, y_{p+1}, \ldots, y_{m}\right)$, is closed. This means that the tensor $\widetilde{\eta}^{t}=t_{1} \widetilde{\eta}_{1}+t_{2} \widetilde{\eta}_{2}$, where $\widetilde{\eta}_{1}=\widetilde{\omega}_{1}^{-1}$ and $\widetilde{\eta}_{2}=\widetilde{\omega}_{2}^{-1}$, determines a nondegenerate Poisson structure in the open subset $U_{L}(x) \subset R_{L}$. Since $\left(t_{1}, t_{2}\right) \notin(\mathbb{R} \times\{0\}) \cup(\{0\} \times \mathbb{R})$, the Poisson structures $\widetilde{\eta}_{1}$ and $\widetilde{\eta}_{2}$ determine a $\widetilde{G}$-invariant bi-Poisson structure on $R_{L}$.

By (13) the bracket on the space $A^{G}$ at the point $x$ induced by the Poisson structure $\eta_{a}(x), a=1,2$, i.e., by the symplectic structure $\omega_{a}(x)$, coincides with the bracket induced by the Poisson structure $\widetilde{\eta}_{a}(x)$. By linearity the brackets on the space $A^{G}$ at the point $x$ induced by Poisson structures $\eta^{t}(x)$ and $\widetilde{\eta}^{t}(x)$ coincide for each $t \in \mathbb{R}^{2}$.

\section{Reduction of a bi-Poisson Structure on the Cotangent Bundle of the Adjoint Orbit of a Compact Lie Group}

In this section we apply our main result (Theorem 1) to some (Kronecker) bi-Poisson structure constructed in our paper [9]. We calculate the reduced bi-Poisson structure on the manifold $R_{H^{0}}$ with the locally free induced action of the group $N\left(H^{0}\right) / H^{0}$ on $R_{H^{0}}$ in the case when $X$ is the cotangent bundle of the adjoint orbit of a compact Lie group $G$.

Let $G$ be a compact connected Lie group with the Lie algebra $\mathfrak{g}$. Denote by $\langle\cdot, \cdot\rangle$ an $\operatorname{Ad}(G)$-invariant scalar product on $\mathfrak{g}$. Let $\mathcal{O} \subset \mathfrak{g}$ be the $\operatorname{Ad}(G)$-orbit through some element $a \in \mathfrak{g}$ of the Lie algebra $\mathfrak{g}$. Then $\mathcal{O}=G / K$, where

$$
K=\{g \in G: \operatorname{Ad}(g)(a)=a\},
$$

is the isotropy group of $a$ (a connected closed subgroup of $G$ ([16], Lemma 5)). Denote by $\Omega$ the canonical symplectic form on the cotangent bundle $T^{*} \mathcal{O}$. The scalar product $\langle\cdot, \cdot\rangle$ determines a $G$-invariant metric on $G / K$. This metric identifies the cotangent bundle $T^{*} \mathcal{O}$ and the tangent bundle $T \mathcal{O}$. Thus we can also talk about the canonical 2-form $\Omega$ on $T \mathcal{O}$. The symplectic form $\Omega$ is $G$-invariant with respect to the natural action of $G$ on $T \mathcal{O}$ (the extension of the action of $G$ on $\mathcal{O}$ ).

Let $\pi: T \mathcal{O} \rightarrow \mathcal{O}$ be the canonical projection. The orbit $\mathcal{O} \subset \mathfrak{g}$ is a symplectic manifold with the Kirillov-Kostant-Souriau form $\omega$ (here we identified the reductive Lie algebra $\mathfrak{g}$ with its dual space $\mathfrak{g}^{*}$ using the invariant scalar product $\langle\cdot, \cdot\rangle$ on $\left.\mathfrak{g}\right)$. Thus we can consider the closed $G$-invariant 2-form $\Omega+\pi^{*} \omega$ on $T \mathcal{O}$. This is a symplectic form on the manifold $X=T \mathcal{O}$ ([9], Prop. 1.6). Put $\omega_{1}=\Omega$ and $\omega_{2}=\Omega+\pi^{*} \omega$. Write $\eta_{1}=\omega_{1}^{-1}, \eta_{2}=\omega_{2}^{-1}$ for the inverse Poisson bi-vectors. The pair of Poisson structures $\left(\eta_{1}, \eta_{2}\right)$ determines a G-invariant bi-Poisson structure $\left\{\eta^{t}=t_{1} \eta_{1}+t_{1} \eta_{1}\right\},\left(t_{1}, t_{2}\right) \in \mathbb{R}^{2}$, on $X$ and the Poisson structure $\eta^{t}$ is degenerate if and only if $t_{1}+t_{2}=0$ ([9], Prop. 1.6).

Let $\hat{G}$ be any connected closed Lie subgroup of $G$ with the Lie algebra $\hat{\mathfrak{g}} \subset \mathfrak{g}$ containing the element $a$. Let $\hat{\mathcal{O}}$ be the adjoint orbit through the element $a \in \hat{\mathfrak{g}}$ in the Lie algebra $\hat{\mathfrak{g}}$. This orbit is a suborbit of $\mathcal{O}$, i.e., $\mathcal{O}=\operatorname{Ad}(\hat{G})(a) \subset \mathcal{O}$. Therefore $\hat{\mathcal{O}}=\hat{G} / \hat{K}$, where $\hat{K}=K \cap \hat{G}$. Denote by $j: T \hat{\mathcal{O}} \rightarrow T \mathcal{O}$ the natural embedding.

Lemma 4. Let $\hat{G}$ be any connected closed Lie subgroup of $G$ with the Lie algebra $\hat{\mathfrak{g}} \subset \mathfrak{g}$ containing the element $a$. The restrictions $\tilde{\omega}_{1}=\left.\omega_{1}\right|_{T \hat{\mathcal{O}}}=j^{*} \omega_{1}$ and $\tilde{\omega}_{2}=\left.\omega_{2}\right|_{T \hat{\mathcal{O}}}=j^{*} \omega_{2}$ are symplectic 


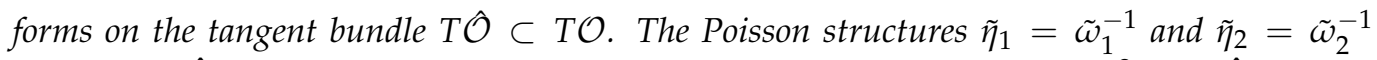

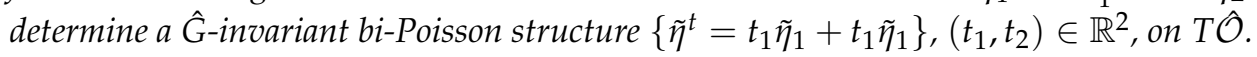

Proof. The restriction of the scalar product $\langle\cdot, \cdot\rangle$ to the subalgebra $\hat{\mathfrak{g}}$ determines a $\hat{G}$ invariant metric on $\hat{G} / \hat{K}$. This metric identifies the cotangent bundle $T^{*} \hat{\mathcal{O}}$ and the tangent bundle $T \hat{\mathcal{O}}$. Denote by $\hat{\Omega}$ the canonical 2 -form on $T \hat{\mathcal{O}}$. By ([17], Prop. 4 ) the canonical form $\hat{\Omega}$ coincides with the restriction $\left.\Omega\right|_{T \hat{\mathcal{O}}}$ of the canonical form $\Omega$, i.e., $\hat{\Omega}=\tilde{\omega}_{1}$.

Identifying the compact Lie algebra $\hat{\mathfrak{g}}$ with its dual space $\hat{\mathfrak{g}}^{*}$ by means of the restriction of the invariant scalar product $\langle\cdot, \cdot\rangle$ to $\hat{\mathfrak{g}}$ we can say about the Kirillov-Kostant-Souriau form $\hat{\omega}$ on the orbit $\hat{\mathcal{O}} \subset \hat{\mathfrak{g}}$. Let us show that $\hat{\omega}=\left.\omega\right|_{\hat{\mathcal{O}}}$.

Indeed, by definition the form $\omega$ is $G$-invariant and at the point $a \in \mathcal{O}$ we have

$$
\omega(a)\left(\left[a, \xi_{1}\right],\left[a, \xi_{2}\right]\right)=-\left\langle a,\left[\xi_{1}, \xi_{2}\right]\right\rangle, \quad \forall \xi_{1}, \xi_{2} \in \mathfrak{g},
$$

where we consider the vectors $\left[a, \xi_{1}\right],\left[a, \xi_{2}\right] \in T_{a} \mathfrak{g}=\mathfrak{g}$ as tangent vectors to the orbit $\mathcal{O} \subset \mathfrak{g}$ at the point $a \in \mathcal{O}$. Since the form $\hat{\omega}$ is described by the similar relation on the Lie algebra $\hat{\mathfrak{g}}$ containing the element $a$, we obtain that $\left.\omega(a)\right|_{T_{a} \mathcal{O}}=\hat{\omega}(a)$. Thus $\hat{\omega}=\left.\omega\right|_{\mathcal{O}}$ by the $\hat{G}$-invariance of the forms $\hat{\omega}$ and $\omega$.

Let $\hat{\pi}: T \hat{\mathcal{O}} \rightarrow \hat{\mathcal{O}}$ be the canonical projection. Consider the closed $\hat{G}$-invariant 2 -form $\hat{\Omega}+\hat{\pi}^{*} \hat{\omega}$ on $T \hat{\mathcal{O}}$. As above, the pair of the $\hat{G}$-invariant symplectic forms $\hat{\omega}_{1}=\hat{\Omega}$ and $\hat{\omega}_{2}=\hat{\Omega}+\hat{\pi}^{*} \hat{\omega}$ on $T \hat{\mathcal{O}}$ determines an $\hat{G}$-invariant bi-Poisson structure by ([9], Prop. 1.6). Taking into account that $\hat{\pi}^{*} \hat{\omega}=\hat{\pi}^{*}\left(\left.\omega\right|_{\mathcal{O}}\right)=\left.\left(\pi^{*} \omega\right)\right|_{T \mathcal{O}}$ and, consequently, $\hat{\omega}_{i}=\tilde{\omega}_{i}, i=1,2$, we complete the proof.

By the lemma above the $G$-invariant bi-Poisson structure $\left\{\eta^{t}=t_{1} \eta_{1}+t_{1} \eta_{1}\right\},\left(t_{1}, t_{2}\right) \in$ $\mathbb{R}^{2}$, on $T \mathcal{O}$ determines the $\hat{G}$-invariant bi-Poisson structure $\left\{\tilde{\eta}^{t}=t_{1} \tilde{\eta}_{1}+t_{1} \tilde{\eta}_{1}\right\},\left(t_{1}, t_{2}\right) \in \mathbb{R}^{2}$, on $T \mathcal{O}$. In general the natural embedding $j: T \mathcal{O} \rightarrow T \mathcal{O}$ is not a Poisson map with respect to the Poisson structures $\tilde{\eta}^{t}$ and $\eta^{t}$, i.e., $j^{*}:\left(\mathcal{E}(T \mathcal{O}),\{,\}_{\eta^{t}}\right) \rightarrow\left(\mathcal{E}(T \mathcal{O}),\{,\}_{\tilde{\eta}^{t}}\right)$ is not a Lie algebra homomorphism. Moreover, the restriction $\left.j^{*}\right|_{A^{G}}:\left(A^{G},\{,\}_{\eta^{t}}\right) \rightarrow\left(\mathcal{E}(T \mathcal{O}),\{,\}_{\tilde{\eta}^{t}}\right)$ to the space $A^{G}$ of the $G$-invariant functions on $T \mathcal{O}$ is not a Lie algebra homomorphism too. However, using Theorem 1 we are able to describe some subgroup $\hat{G} \subset G$ and the corresponding orbit $\hat{\mathcal{O}}=\hat{G} / \hat{K}$ for which the map $\left.j^{*}\right|_{A^{G}}$ is a Lie algebra homomorphism (for any $t$ ), its image lies in the space $A^{\hat{G}}$ of $\hat{G}$-invariant functions on $T \hat{\mathcal{O}}$ and the action of the group $\hat{G} / C(\hat{G})$ on $T \hat{\mathcal{O}}$ is locally free (see Proposition 1 below). Here $C(\hat{G})$ stands for the center of the Lie group $\hat{G}$ (which is the kernel of the adjoint representation of $\hat{G}$ ).

Let us describe the corresponding subgroups starting from the subgroup $H \subset G$ determining the principal orbit type submanifold $X_{(H)}$ of the $G$-manifold $X=T(G / K)$. As we remarked above in this case the manifold $X_{(H)}$ is a connected open dense subset of $X$. Denote by $\mathfrak{k}$ the Lie algebra of $K$ and by $\mathfrak{m}$ the orthogonal complement to $\mathfrak{k}$ in $\mathfrak{g}$ with respect to the form $\langle\cdot, \cdot\rangle$. Taking into account that $G$ acts on the base $\mathcal{O} \subset \mathfrak{g}$ transitively and identifying the tangent space $T_{0} \mathcal{O}$ at $o=K$ with the space $\mathfrak{m}$, we obtain that

$$
H=\left\{k \in K: \operatorname{Ad}(k)\left(x_{0}\right)=x_{0}\right\}=K_{x_{0}},
$$

for some $x_{0} \in \mathfrak{m}$ such that the centralizer $\mathfrak{k}_{x_{0}} \stackrel{\text { def }}{=}\left\{y \in \mathfrak{k}:\left[x_{0}, y\right]=0\right\}$ has the minimal possible dimension. It is clear that the Lie algebra $\mathfrak{h}$ of $H$ coincides with the Lie algebra $\mathfrak{k}_{x_{0}}$. Consider the compact Lie subalgebra

$$
\hat{\mathfrak{g}}=\left\{y \in \mathfrak{g}:[y, z]=0, \forall z \in \mathfrak{h}=\mathfrak{k}_{x_{0}}\right\},
$$

of $\mathfrak{g}$. Denote by $\hat{G}$ the connected Lie subgroup of $G$ with the Lie algebra $\hat{\mathfrak{g}}$. The Lie group $\hat{G}$ is closed in $G$ because $\hat{G}$ is the identity component of the centralizer of $H^{0}$ in $G$. Moreover, $a$ is an element of $\hat{\mathfrak{g}}$ because by definition $[a, \mathfrak{k}]=0$ and $\mathfrak{h} \subset \mathfrak{k}$. Thus, as above, we can consider $\operatorname{Ad}(\hat{G})$-suborbit $\hat{\mathcal{O}} \subset \hat{\mathfrak{g}}$ of the orbit $\mathcal{O}$ through the element $a$ and the natural embedding $j: T \hat{\mathcal{O}} \rightarrow T \mathcal{O}$. 
Proposition 1. Let $\hat{G}$ be the connected Lie subgroup of $G$ with the Lie algebra $\hat{\mathfrak{g}}$ defined by (16). Then

(1) The restrictions $\tilde{\omega}_{1}=\left.\omega_{1}\right|_{T \mathcal{O}}=j^{*} \omega_{1}$ and $\tilde{\omega}_{2}=\left.\omega_{2}\right|_{T \mathcal{O}}=j^{*} \omega_{2}$ are symplectic forms on the tangent bundle $\mathrm{TO} \subset \mathrm{TO}$;

(2) The Poisson structures $\tilde{\eta}_{1}=\tilde{\omega}_{1}^{-1}$ and $\tilde{\eta}_{2}=\tilde{\omega}_{2}^{-1}$ determine a $\hat{G}$-invariant bi-Poisson structure $\left\{\tilde{\eta}^{t}=t_{1} \tilde{\eta}_{1}+t_{2} \tilde{\eta}_{2}\right\},\left(t_{1}, t_{2}\right) \in \mathbb{R}^{2}$, on $\mathrm{T} \mathcal{O}$;

(3) For any $t \in \mathbb{R}^{2}$ the map $j^{*}$ is a Poisson map of the $\eta^{t}$-Poisson algebra $A^{G}$ of the G-invariant functions on $\mathrm{TO}$ into the $\tilde{\eta}^{t}$-Poisson algebra $A^{\hat{G}}$ of the $\hat{G}$-invariant function on $\mathrm{T} \hat{\mathcal{O}}$;

(4) The action of the Lie group $\hat{G} / C(\hat{G})$ on $T \mathcal{O}$ is locally free $(C(\hat{G})$ is the center of $\hat{G})$;

(5) The map $j^{*}: A^{G} \rightarrow A^{\hat{G}}$ is an injection and the image $j^{*}\left(A^{G}\right)$ functionally generates the space $A^{\hat{G}}$.

Proof. Items (1) and (2) follow immediately from Lemma 4. To prove items (3)-(5) we will describe the submanifolds $R_{H}=X_{H}$ and $R_{H^{0}}$ of $X_{(H)}$ defined by relations (2), (6) and will show that some connected component of $R_{H^{0}}$ is open and dense in $T \mathcal{O}$. To this end we will use some calculation from the paper ([9], ((Sections 2.1 and 3.3)).

It is clear that $\hat{\mathcal{O}}=\hat{G} / \hat{K}$, where $\hat{K}=\hat{G} \cap K$. Since the form $\langle\cdot, \cdot\rangle$ is $\operatorname{Ad}(G)$-invariant, we have $[\mathfrak{k}, \mathfrak{m}] \subset \mathfrak{m}$ and $\operatorname{ad}\left(x_{0}\right)(\mathfrak{k}) \subset \mathfrak{m}$, where $x_{0}$ is that mentioned in Formula (15). Let

$$
\mathfrak{m}\left(x_{0}\right)=\left\{y \in \mathfrak{m}:\left\langle y, \operatorname{ad}\left(x_{0}\right)(\mathfrak{k})\right\rangle=0\right\} .
$$

By the $\operatorname{Ad}(G)$-invariance of $\langle\cdot, \cdot\rangle$, we have that $x_{0} \in \mathfrak{m}\left(x_{0}\right)$. The Lie group $K$ is compact, hence by Remark 1 below,

$$
\operatorname{Ad}(K)\left(\mathfrak{m}\left(x_{0}\right)\right)=\mathfrak{m} \quad \text { and, consequently, } G \cdot\left(\mathfrak{m}\left(x_{0}\right)\right)=T(G / K),
$$

i.e., each $G$-orbit in $T(G / K)$ intersects the linear subspace $\mathfrak{m}\left(x_{0}\right) \subset \mathfrak{m}=T_{o}(G / K)$.

Remark 1. Relations (17) hold if $x_{0}$ is replaced by an arbitrary element $x \in \mathfrak{m}$. This follows easily from the fact that for any (fixed) $y \in \mathfrak{m}$ the function $k \mapsto\langle y, \operatorname{Ad}(k)(x)\rangle$ on the compact group $K$ attains its maximum value at some point $k_{y} \in K$. Differentiating $\left\langle y, \operatorname{Ad}\left(k_{y} \exp t \xi\right)(x)\right\rangle$ with $\xi \in \mathfrak{k}$, we obtain that $\operatorname{Ad}\left(k_{y}^{-1}\right)(y) \perp \operatorname{ad}(x)(\mathfrak{k})$.

Consider the $\operatorname{Ad}(K)$-action of the compact Lie group $K$ on $\mathfrak{m}$. The space $\mathfrak{m}\left(x_{0}\right)$ is the orthogonal complement to the tangent space $T_{x_{0}}\left(\operatorname{Ad}(K)\left(x_{0}\right)\right)=\operatorname{ad}\left(x_{0}\right)(\mathfrak{k})$ of the orbit $\operatorname{Ad}(K)\left(x_{0}\right) \subset \mathfrak{m}$ at $x_{0}$ in $\mathfrak{m}$ ([12], Th.2.3.28). Hence some open neighborhood of $x_{0}$ in the linear space $\mathfrak{m}\left(x_{0}\right) \ni x_{0}$ is a slice for $\operatorname{Ad}(K)$-action at $x_{0}$. Since the group $H=K_{x_{0}}$ represents the principal orbit type, the action of $H$ on this open neighborhood of $x_{0}$ and, consequently, on the whole linear space $\mathfrak{m}\left(x_{0}\right)$ is trivial, i.e.,

$$
\operatorname{Ad}(h)(x)=x \quad \text { for all } h \in H \text { and } x \in \mathfrak{m}\left(x_{0}\right),
$$

and, consequently,

$$
\left[\mathfrak{m}\left(x_{0}\right), \mathfrak{h}\right]=0,
$$

(see ([18], Prop. 9) for another proof of identity (19)). It is clear that $\mathfrak{m}\left(x_{0}\right) \cap X_{H}$ is an open dense subset of $\mathfrak{m}\left(x_{0}\right)$. Let $R_{H^{0}}$ be the submanifold of the connected manifold $X_{(H)}$ defined by relation (6) for $L=H^{0}$. From (18) and the definitions of the manifolds $X_{(H)}, X_{H}$ and $R_{H^{0}}$ it follows easily that

$$
\mathfrak{m}\left(x_{0}\right) \cap X_{H}=\mathfrak{m}\left(x_{0}\right) \cap X_{(H)}=\mathfrak{m}\left(x_{0}\right) \cap R_{H^{0}} .
$$

Let us show that

$$
X_{H}=N(H) \cdot\left(\mathfrak{m}\left(x_{0}\right) \cap X_{H}\right) \quad \text { and } \quad R_{H^{0}}=N\left(H^{0}\right) \cdot\left(\mathfrak{m}\left(x_{0}\right) \cap R_{H^{0}}\right),
$$


where $N(H)$ is the normalizer of $H$ in $G$ and $N\left(H^{0}\right)$ is the normalizer of the identity component $H^{0}$ of $H$ in $G$. Indeed, by (17) each point of the manifold $X_{H}$ has the form $g \cdot x$ for some $g \in G, x \in \mathfrak{m}\left(x_{0}\right)$ and for this point $G_{g \cdot x}=H$. However, $G_{g \cdot x}=g G_{x} g^{-1}$ and by (18) $H \subset G_{x}$. Therefore $g H^{-1} \subset H$. Since $g \mathrm{Hg}^{-1}$ is an open subgroup of $H$ and the compact group $H$ has a finite number of connected component, $g \mathrm{Hg}^{-1}=H$, i.e., $g \in N(H)$. Similarly, each point of the manifold $R_{H^{0}}$ has the form $g \cdot x$ for some $g \in G$, $x \in \mathfrak{m}\left(x_{0}\right)$ and for this point $\left(G_{g \cdot x}\right)^{0}=H^{0}$. Also $G_{g} \cdot x=g G_{x} g^{-1}$ and by (18) $H \subset G_{x}$. Then $H^{0}=g G_{x}^{0} g^{-1} \subset G_{x}^{0}$, and, consequently, $H^{0}=g G_{x}^{0} g^{-1}=G_{x}^{0}$. Thus $g H^{0} g^{-1}=H^{0}$, i.e., $g \in N\left(H^{0}\right)$.

Note that the subgroup $N\left(H^{0}\right)$ of $G$ is closed (compact) and therefore contains only a finite number of connected components, i.e., $\left|N\left(H^{0}\right) /\left(N\left(H^{0}\right)\right)^{0}\right|<\infty$. Since by Lemma 1 $R_{H^{0}}$ is an embedded submanifold of $X_{(H)}$ and of $X=T(G / K)$, its connected component $R_{H^{0}}^{x_{0}}$ containing $x_{0}$ has the form

$$
R_{H^{0}}^{x_{0}}=\left(N\left(H^{0}\right)\right)^{0} \cdot\left(\mathfrak{m}\left(x_{0}\right) \cap R_{H^{0}}\right),
$$

and (see (8))

$$
\mathbf{X}=X_{(H)} / G \simeq R_{H^{0}} /\left(N\left(H^{0}\right) / H^{0}\right) \simeq R_{H^{0}}^{x_{0}} /\left(\left(N\left(H^{0}\right)\right)^{x_{0}} / H^{0}\right),
$$

where $\left(\left(N\left(H^{0}\right)\right)^{x_{0}}\right.$ is the normalizer of the component $R_{H^{0}}^{x_{0}}$ in the group $N\left(H^{0}\right)$ (containing the connected component $\left(\left(N\left(H^{0}\right)\right)^{0}\right.$ of $\left.N\left(H^{0}\right)\right)$. Since by definition $H \subset N\left(H^{0}\right)$, we see that $h \cdot\left(N\left(H^{0}\right)\right)^{0} \cdot h^{-1}=\left(N\left(H^{0}\right)\right)^{0}$ for any $h \in H$. Taking into account that $\operatorname{Ad}(H)(x)=x$ for each $x \in \mathfrak{m}\left(x_{0}\right)$, we obtain that

$$
H \subset\left(N\left(H^{0}\right)\right)^{x_{0}} .
$$

Now it is clear that the manifold $R_{H^{0}}^{x_{0}}$ is a single orbit type $\left(N\left(H^{0}\right)\right)^{x_{0}} / H^{0}$-manifold with a discrete isotropy group isomorphic to $H / H^{0}$ (the group $H^{0}$ acts trivially on $R_{H^{0}}^{x_{0}}$ ).

We will show that the connected component $R_{H^{0}}^{x_{0}}$ of the manifold $R_{H^{0}}$ containing the element $x_{0}$ is an open dense subset of $T \hat{\mathcal{O}}$. To this end consider the subalgebra $\hat{\mathfrak{k}}=\mathfrak{k} \cap \hat{\mathfrak{g}}$ of $\mathfrak{k}$. Since $\mathfrak{k}$ is the centralizer of $a \in \mathfrak{g}$ in $\mathfrak{g}$, the element $a \in \mathfrak{k}$ belongs to $\hat{\mathfrak{k}}\left(\left[a, \mathfrak{k}_{x_{0}}\right]=[a, \mathfrak{k}]=0\right)$. Denote by $\hat{\mathfrak{m}}$ the orthogonal complement to $\hat{\mathfrak{k}}$ in $\hat{\mathfrak{g}}$ with respect to the form $\left.\langle\cdot, \cdot\rangle\right|_{\hat{\mathfrak{g}}}$. By (19) $\mathfrak{m}\left(x_{0}\right) \subset \hat{\mathfrak{m}}$. Moreover, $\mathfrak{m}\left(x_{0}\right)$ is the orthogonal complement of the space $\operatorname{ad}\left(x_{0}\right)(\hat{\mathfrak{k}})$ in $\hat{\mathfrak{m}}$ ([9], Prop. 2.3), i.e., $\hat{\mathfrak{m}}\left(x_{0}\right)=\mathfrak{m}\left(x_{0}\right)$. Now applying Remark 1 to the pair $(\hat{G}, \hat{K})$ we get $\hat{G} \cdot \mathfrak{m}\left(x_{0}\right)=T \hat{\mathcal{O}}$.

Since $\hat{G}$ is a connected component of the centralizer of $H^{0}$ in $G$, we have that $g h=h g$ for all elements $g \in \hat{G}$ and $h \in H^{0}$. Since the compact Lie algebra $\mathfrak{h}$ is reductive, we have that $\mathfrak{n}(\mathfrak{h})=\hat{\mathfrak{g}}+\mathfrak{h}$ for the normalizer $\mathfrak{n}(\mathfrak{h})$ of $\mathfrak{h}$ in $\mathfrak{g}$. However, $\mathfrak{n}(\mathfrak{h})=\mathfrak{n}\left(H^{0}\right)$ and thus $\hat{G} \cdot H^{0} \subset G$ is the identity component of the normalizer $N\left(H^{0}\right)$. However, $H^{0} \cdot \mathfrak{m}\left(x_{0}\right)=$ $\mathfrak{m}\left(x_{0}\right)$ by $(18)$, and therefore

$$
\begin{aligned}
T \hat{\mathcal{O}} & =\hat{G} \cdot \mathfrak{m}\left(x_{0}\right)=\left(\hat{G} \cdot H^{0}\right) \cdot \mathfrak{m}\left(x_{0}\right) \\
& =\left(N\left(H^{0}\right)\right)^{0} \cdot \mathfrak{m}\left(x_{0}\right)=\left(N\left(H^{0}\right)\right)^{x_{0}} \cdot \mathfrak{m}\left(x_{0}\right) .
\end{aligned}
$$

Since by (22) $\left(N\left(H^{0}\right)\right)^{0} \cdot\left(\mathfrak{m}\left(x_{0}\right) \cap R_{H^{0}}\right)$ is the connected component $R_{H^{0}}^{x_{0}}$ of the manifold $R_{H^{0}}, R_{H^{0}}^{x_{0}}$ is an open dense subset of $\hat{X}=T \hat{\mathcal{O}}$. This subset is $\hat{G}$-invariant because $\hat{G} \subset\left(N\left(H^{0}\right)\right)^{0}$. However, $X_{(H)} \subset T \mathcal{O}$ and $R_{H^{0}}^{x_{0}} \subset T \hat{\mathcal{O}}$. Thus by (23) and Theorem 1 for any $t \in \mathbb{R}^{2}$ the map $i^{*}=\left(\left.j\right|_{R_{H^{0}}^{x_{0}}}\right)^{*}$ is a Poisson map of the $\eta^{t}$-Poisson algebra of the $G$-invariant functions on $X_{(H)}$ into the $\tilde{\eta}^{t}$-Poisson algebra of the $\left(N\left(H^{0}\right)\right)^{x_{0}} / H^{0}$-invariant functions on $R_{H^{0}}^{x_{0}}$. Now to prove item (3) it is sufficient to remark that $X_{(H)}$ and $R_{H^{0}}^{x_{0}}$ are open and dense in $T \mathcal{O}$ and $T \hat{\mathcal{O}}$, respectively. 
By Lemma 1 the action of $\left(N\left(H^{0}\right)\right) / H^{0}$ on $R_{H^{0}}$ is locally free. Thus the actions of the groups $\left(N\left(H^{0}\right)\right)^{x_{0}} / H^{0}$ and $\left(N\left(H^{0}\right)\right)^{0} / H^{0}$ on $R_{H^{0}}^{x_{0}}$ are also locally free. As we remarked above $R_{H^{0}}^{x_{0}}$ is a single orbit type $\left(N\left(H^{0}\right)\right)^{x_{0}} / H^{0}$-manifold with a discrete isotropy group isomorphic to $H / H^{0}$. Therefore by (24) $R_{H^{0}}^{x_{0}}$ is also a single orbit type $\hat{G}$-manifold with the isotropy group isomorphic to $\hat{H}=\hat{G} \cap H$ and $\hat{H}$ is a Lie group determining the principal orbit type for the $\hat{G}$-action on $T \hat{\mathcal{O}}$. Taking into account that $[\hat{\mathfrak{g}}, \mathfrak{h}]=0$ by definition, we obtain that the Lie algebra $\hat{\mathfrak{g}} \cap \mathfrak{h}$ is a subalgebra of the center of $\hat{\mathfrak{g}}$ and, consequently, $\hat{G} \cap H^{0} \subset C(\hat{G})$, where $C(\hat{G})$ is the kernel of the adjoint representation of $\hat{G}$. Thus $\hat{G} \cap H^{0} \subset$ $C(\hat{G}) \cap H$. Therefore the action of the group $\hat{G} / C(\hat{G})$ on $R_{H^{0}}^{x_{0}} \subset T \hat{\mathcal{O}}$ with a discrete isotropy group isomorphic to some quotient group of $(\hat{G} \cap H) /\left(\hat{G} \cap H^{0}\right)$ is locally free, item (4) is proved.

Since $\left(N\left(H^{0}\right)\right)^{0}=\hat{G} \cdot H^{0}$ and $H^{0}$ acts trivially on $R_{H^{0}}^{x_{0}}$, each connected component of the $\left(N\left(H^{0}\right)\right)^{x_{0}}$-orbit in $R_{H^{0}}^{x_{0}}$ is some $\hat{G}$-orbit and, consequently, the natural projection $R_{H^{0}}^{x_{0}} / \hat{G} \rightarrow R_{H^{0}}^{x_{0}} /\left(N\left(H^{0}\right)\right)^{x_{0}}$ is a covering. Taking into account that $X_{(H)} / G \simeq$ $R_{H^{0}}^{x_{0}} /\left(N\left(H^{0}\right)\right)^{x_{0}}$ (see (23)) we complete the proof of (5).

Funding: This research received no external funding.

Institutional Review Board Statement: Not applicable.

Informed Consent Statement: Not applicable.

Data Availability Statement: Not applicable.

Conflicts of Interest: The author declares no conflict of interest.

\section{Appendix A}

The goal of this section is to provide the reader with a proof of a statement that is well known to experts but does not seem to be readily available in the literature.

Let $G$ be a connected Lie group and $L$ be its closed subgroup. Denote by $\mathfrak{g}$ and $\mathfrak{l}$ the Lie algebras of $G$ and $L$, respectively. Let $N(L)$ be the normalizer group of $L$ in $G$ and let $\mathfrak{n}(L)$ be its Lie algebra. It is clear that $\mathfrak{l} \subset \mathfrak{n}(L)$ because $L \subset N(L)$.

Lemma A1. Let $\alpha, \beta$ be two $\operatorname{Ad}(L)$-invariant scalar products on the algebra Lie $\mathfrak{g}$. Let $\mathfrak{p}^{\alpha}$ and $\mathfrak{p}^{\beta}$ be the orthogonal complements to $\mathfrak{n}(L)$ in $\mathfrak{g}$ with respect to the forms $\alpha$ and $\beta$ respectively. Then

$$
\mathfrak{p}^{\alpha} \oplus \mathfrak{l}=\mathfrak{p}^{\beta} \oplus \mathfrak{l} .
$$

Proof. The action of the subgroup $\operatorname{Ad}(L) \subset \operatorname{Ad}(G)$ on $\mathfrak{g}$ determines naturally the action $\widetilde{\text { Ad }}$ of the group $L$ on $\mathfrak{g} / \mathfrak{l}$ because $\operatorname{Ad}(L)(\mathfrak{l})=\mathfrak{l}$. Additionally, the forms $\alpha, \beta$ determine the $\widetilde{\mathrm{Ad}}(L)$-invariant scalar products on the quotient space $\mathfrak{g} / \mathfrak{l}$ which we denote by $\alpha^{\prime}$ and $\beta^{\prime}$ respectively. Let $\pi: \mathfrak{g} \rightarrow \mathfrak{g} / \mathrm{l}$ be the natural projection. By definition, the spaces $\pi\left(\mathfrak{p}^{\alpha}\right)$ and $\pi\left(\mathfrak{p}^{\beta}\right)$ are the orthogonal complements to the space $\pi(\mathfrak{n}(L))$ in $\mathfrak{g} / \mathfrak{l}$ with respect to the forms $\alpha^{\prime}$ and $\beta^{\prime}$, respectively. Since the scalar product $\alpha^{\prime}$ on $\mathfrak{g} / \mathfrak{l}$ is $\widetilde{\mathrm{Ad}}(L)$-invariant, there exists a unique nondegenerate linear map $J: \mathfrak{g} / \mathfrak{l} \rightarrow \mathfrak{g} / \mathfrak{l}$ such that $\beta^{\prime}(u, v)=\alpha^{\prime}(u, J v)$ for all $u, v \in \mathfrak{g} / \mathfrak{l}$ and $J \cdot \widetilde{\operatorname{Ad}}(h)=\widetilde{\operatorname{Ad}}(h) \cdot J$ for all $h \in L$. But by ([12], Lemma 2.1.13) the Lie algebra $\mathfrak{n}(L)$ of the normalizer group $N(L)$ of closed subgroup $L$ in $G$ is determined by the following equation

$$
\mathfrak{n}(L)=\{\xi \in \mathfrak{g}: \operatorname{Ad}(h)(\xi)-\xi \in \mathfrak{l}, \forall h \in L\},
$$

and therefore

$$
\pi(\mathfrak{n}(L))=\mathfrak{n}(L) / \mathfrak{l}=\{v \in \mathfrak{g} / \mathfrak{l}: \widetilde{\operatorname{Ad}}(h)(v)=v, \forall h \in L\} .
$$


Now we get the inclusion $J(\pi(\mathfrak{n}(L))) \subset \pi(\mathfrak{n}(L))$ due to the fact that $J$ commutes with the $\widetilde{\operatorname{Ad}}(L)$-action on $\mathfrak{g} / \mathfrak{l}$ and $\pi(\mathfrak{n}(L)) \subset \mathfrak{g} / \mathfrak{l}$ is the set of all $\widetilde{\operatorname{Ad}}(L)$-fixed vectors in $\mathfrak{g} / \mathfrak{l}$. Therefore

$$
\beta^{\prime}\left(\pi\left(\mathfrak{p}^{\alpha}\right), \pi(\mathfrak{n}(L))\right)=\alpha^{\prime}\left(\pi\left(\mathfrak{p}^{\alpha}\right), J(\pi(\mathfrak{n}(L)))=\alpha^{\prime}\left(\pi\left(\mathfrak{p}^{\alpha}\right), \pi(\mathfrak{n}(L))\right)=0,\right.
$$

and, consequently, $\pi\left(\mathfrak{p}^{\alpha}\right)=\pi\left(\mathfrak{p}^{\beta}\right)$, i.e. $\mathfrak{p}^{\alpha} \oplus \mathfrak{l}=\mathfrak{p}^{\beta} \oplus \mathfrak{l}$.

\section{References}

1. Magri, F. A simple model of the integrable hamiltonian equation. J. Math. Phys. 1978, 19, 1156-1162. [CrossRef]

2. Olver, P. Applications of Lie Groups to Differential Equations; Springer: Berlin/Heidelberg, Germany, 1986.

3. Gelfand, I.; Zakharevich, I.On the Local Geometry of a Bihamiltonian Structure. In The Gelfand Mathematical Seminars 1990-1992; Birkhauser: Basel, Switzerland, 1993; pp. 51-112.

4. Bolsinov, A. Compatible Poisson brackets on Lie algebras and completeness of families of functions in involution. Math. USSR Izv. 1992, 38, 69-90. (In Russian) [CrossRef]

5. Bolsinov, A.; Oshemkov, A.Bi-hamiltonian structures and singularities of integrable Hamiltonian systems. Regul. Chaotic Dyn. 2009, 14, 431-454. [CrossRef]

6. Gelfand, I.; Zakharevich, I. Webs, Lenard schemes, and the local geometry of bihamiltonian Toda and Lax structures. Sel. Math. 2000, 6, 131-183. [CrossRef]

7. Panasyuk, A. Projections of jordan bi-poisson structures that are kronecker, diagonal actions, and the classical gaudin systems. J. Geom. Phys. 2003, 47, 379-397. [CrossRef]

8. Guillemin, V.; Sternberg, S. Convexity properties of the moment map. Invent. Math. 1982, 67, 491-513. [CrossRef]

9. Mykytyuk, I.; Panasyuk, A. Bi-poisson structures and integrability of geodesic flow on homogeneous spaces. Transform. Groups 2004, 9, 289-308. [CrossRef]

10. Marsden, J.; Ratiu, T. Introduction to Mechanics and Symmetry; Springer: Berlin, Germany, 1999.

11. Duistermaat, J.; Kolk, J. Lie Groups, Universitext; Springer: Berlin/Heidelberg, Germany, 2000.

12. Ortega, J.; Ratiu, T. Momentum Maps and Hamiltonian Reduction, Volume 222 of Program Mathematics; Birkhauser Boston, Inc.: Boston, MA, USA, 2004.

13. Mykytyuk, I.; Panasyuk, A. Dirac brackets and reduction of invariant bi-Poisson structures. arXiv 2016, arXiv:1605.03382.

14. Bourbaki, N. Groupes et Algèbres de Lie, I-III, Éléments de Mathématique; Hermann: Paris, France, 1971.

15. Guillemin, V.; Sternberg, S. Symplectic Techniques in Physics; Cambridge University Press: Cambridge, UK, 1984.

16. Kostant, B. Lie group representation on polynomial rings. Am. J. Math. 1963, 85, 327-404. [CrossRef]

17. Mykytyuk, I. Integrability of geodesic flows for metrics on suborbits of the adjoint orbits of compact groups. Transform. Groups 2016, 21, 531-553. [CrossRef]

18. Mykytyuk, I. Actions of borel subgroups on homogeneous spaces of reductive complex lie groups and integrability. Compos. Math. 2001, 127, 55-67. [CrossRef] 